\title{
COVID-19 y personas mayores: representaciones sociales en el Ministerio de Salud de Chile ${ }^{1}$
}

\section{Carola Salazar-Norambuena}

(iD) https://orcid.org/0000-0001-9056-5926

Universidad Católica de la Santísima Concepción, Chile csalazar@ucsc.cl

RESUMEN

Las representaciones sociales son un conjunto de ideas, prácticas e ideales que forman parte del sentido común de un grupo. Por lo general, el estudio de las representaciones de las personas mayores se realiza desde los medios de comunicación; sin embargo, esta investigación incorpora a un agente clave en los procesos de salud/ enfermedad/atención: los ministerios de Salud. El objetivo de este artículo es identificar la representación de las personas mayores en las noticias del sitio web del Ministerio de Salud Chileno (MINSAL), en el contexto de pandemia por COVID-19, durante el año 2020. Utilizando un análisis de «marcos» (frames), se encontraron cuatro tipos: subgrupos etarios; términos para referirse a las personas mayores; grupo de riesgo y solidaridad social. Estos cuatro marcos comparten las cualidades de ser viejistas, es decir, que tienen una visión sesgada de las personas mayores, representándolas como un grupo homogéneo, dependiente y sin agencia, pues son otras personas las que hablan por ellas.

Palabras clave: personas mayores, Chile, viejismo, COVID-19, frames.

\footnotetext{
1 El financiamiento fue otorgado por la Universidad Católica de la Santísima Concepción, FAA código 02/2019.
} 


\section{COVID-19 and older adults: Representations from the Chilean Health Ministry}

ABSTRACT

Social representations are a set of ideas, practices and ideals that are part of the common sense of a group. Generally, the study of older adults'representations is conducted by looking at the media, however, this research incorporates a key agent in the health/ disease /care processes: the Ministry of Health. The main objective of this article is to identify older adults' representations found in the Chilean Ministry of Health (MINSAL) news portal website during the COVID-19 pandemic in 2020. Using framing analysis, four types of frames were found: age sub-groups, terms for referring to older adults, risk group and social solidarity. These four frames share some ageist qualities, that is, they have a biased view of older people, representing them as a homogeneous, dependent and without agency group, since other people speak for them.

Keywords: Older adults, Chile, Ageism, COVID-19, Framing analysis. 


\section{INTRODUCCIÓN}

La pandemia provocada por el virus Sars-CoV-2, ha afectado de forma considerable a países del mundo entero, afectando no solo aspectos biológicos y sanitarios, sino también socioculturales. Desde esta premisa, surge la importancia de considerar los aspectos mediáticos de estos eventos, porque los diferentes discursos que se generan en torno a estas experiencias cumplen un rol crucial en la creación de los significados que se dan a estos (Nespereira, 2014).

A pesar de que la enfermedad provocada por el Sars-CoV-2, denominada COVID-19, puede presentarse en cualquier grupo etario, el grupo de personas mayores de sesenta años ha estado en la palestra, puesto que una evidencia preliminar sugiere que, en dieciséis países examinados, las personas de este grupo presentan mayores tasas de mortalidad por esta enfermedad en comparación a grupos más jóvenes, sobre todo por la presencia de comorbilidades (Kang y Jung, 2020; Shahid et al., 2020; Yañez, Weiss, Romand y Treggiari, 2020). Esto ha provocado «un brote paralelo de discriminación por edad» en el mundo, que se caracteriza por homogeneizar a las personas mayores como un grupo frágil, vulnerable y que necesita ser auxiliado (Ayalon et al., 2020), omitiendo la importancia de los contextos socioculturales, territoriales, genéticos y personales en la experiencia de los procesos de salud/enfermedad/atención (también conocido como s/e/a). Menéndez (2009) propone considerar como una triada estos procesos, porque tanto las percepciones de salud, como de enfermedad y el tipo de atención están altamente relacionados. De esta forma, las prácticas para entender, enfrentar y solucionar los procesos de salud/enfermedad/atención dependen del contexto histórico-sociocultural y los significados que la persona que lo padece le atribuye. Por tanto, para comprender cómo diferentes personas (e instituciones) viven el COVID-19, es importante considerar el proceso de s/e/a completo, considerando las «causales específicas de los padecimientos, las formas de atención y los sistemas ideológicos (significados) respecto de los mismos» (Menéndez, 1994, p. 72). 
Otro aspecto a considerar es el aumento de la población de personas de sesenta años y más durante las últimas décadas en América Latina y el Caribe. Sin embargo, la situación en la región es heterogénea: mientras algunos países presentan niveles avanzados o muy avanzados de envejecimiento, otros aún se encuentran en una etapa incipiente (Cepal, 2020). De esta forma, se vuelve importante conocer las diferentes realidades de cada país, para evitar las generalizaciones, sobre todo en torno a los discursos que surgen en torno a este grupo etario en el contexto de pandemia.

Dentro de estos discursos, surgen las representaciones sociales. Moscovici (2008) las define como sistemas de valores, ideas y prácticas compartidas por una comunidad. De esta forma, las representaciones permiten los miembros de una sociedad comprender, explicar y dominar distintos aspectos de la vida diaria, porque son elaboradas colectivamente y se recrean constantemente.

Si bien gran parte de los estudios sobre representaciones de las personas mayores se basan los medios de comunicación (Zulman et al., 2011) esta investigación busca incluir a otro agente clave en la representación de este grupo: el Ministerio de Salud. Considerando que las experiencias, expectativas y comportamientos de las personas mayores son influenciadas por los mensajes que expresan los medios de comunicación, los profesionales de la salud y la familia, entre otros (Ayalon y Tesch-Römer, 2018), y el rol crucial que cumplen los ministerios de Salud en la comunicación del riesgo y el manejo/prevención de los procesos de salud y enfermedad (Rahim, Ibrahim, Salim e Ikram, 2019), este artículo se centra en el análisis de las noticias que fueron publicadas en el sitio web del Ministerio de Salud de Chile (MINSAL) durante la pandemia del COVID-19 en el año 2020, enfocándose en la representación de las personas mayores, que en el caso de Chile, según la Ley N ${ }^{\circ} 19.828$ de 2002, corresponde a las personas desde los sesenta años de edad.

El artículo se divide en cuatro secciones: primero, se contextualizará cómo el COVID-19 ha afectado a la población chilena desde marzo de 2020, cuando fue diagnosticado el primer caso por esta enfermedad, y las principales medidas que adoptó el gobierno chileno durante ese año. Luego, se presentan las aproximaciones teóricas: primero la relación existente en las emergencias sanitarias entre autoridades y medios de comunicación masivos. En segundo lugar, se describe el concepto de representaciones sociales de Moscovici, y el espacio donde estas se generan: la esfera pública. Luego, están los conceptos relacionados con el análisis de contenido de las noticias: la forma en que las personas mayores han sido representadas en los medios de comunicación, y el análisis por 
marcos (framing analysis). Después, se presenta el marco metodológico de la investigación, centrada en el análisis de contenido de las noticias del sitio web del Ministerio de Salud de Chile, para luego continuar con los principales resultados. Finalmente, la discusión y principales conclusiones abordan aspectos claves que nacen del análisis realizado.

\section{COVID-19 EN CHILE}

El 3 de marzo de 2020, el Ministerio de Salud confirmó el primer caso de COVID-19 en el país. Se trataba de un hombre de 33 años, quien había viajado durante un mes por diferentes países del sudeste asiático (MINSAL, 2020a). Los siguientes casos diagnosticados correspondían a personas que también habían realizado viajes internacionales. Sin embargo, semanas más tarde —el 14 de marzo - el ministerio reconoce que los casos diagnosticados de COVID-19 ya no pueden ser trazados a viajeros internacionales, por lo que pasó a fase 3 de contagio. Esa misma noche, el presidente de la República, Sebastián Piñera, anunció a través de cadena nacional las principales medidas que el gobierno tomaría para enfrentar el potencial aumento de contagios.

En términos generales, a lo largo del año 2020, el gobierno chileno implementó diversas estrategias; una de ellas fue el Plan Paso a Paso, que es «una estrategia gradual para enfrentar la pandemia según la situación sanitaria de cada zona en particular» (Gobierno de Chile, 2021a). Este plan consiste en cuatro pasos, que van desde el número 1, con mayores restricciones, cuarentena, hasta el paso 4, apertura inicial.

Mientras este plan estaba orientado a la población general, otras estrategias se concentraron específicamente en las personas mayores, que fue un grupo considerado desde el inicio como de riesgo (MINSAL, 2020b). Si bien las personas desde que cumplen los sesenta años son consideradas legalmente en el país como «adultos mayores» (Ley $\mathrm{N}^{\circ}$ 19.818), para efectos de las medidas, la edad de corte variaba. Por ejemplo, una de las primeras medidas anunciadas el 14 de marzo de 2020 prohibió el funcionamiento de clubes y centros de día de adultos mayores y las visitas a los establecimientos de larga estadía para adultos mayores (ELEAM); en ambos grupos, pueden participar personas desde los sesenta años. Paralelamente, todos los funcionarios públicos que tenían desde 75 años podían realizar su trabajo de forma telemática si así lo deseaban.

Otra medida fue la creación de residencias sanitarias paras las personas enfermas de COVID-19 que necesitaban estar aisladas, con un principal enfoque 
en las personas mayores. Luego, el 22 de marzo se estableció una cuarentena preventiva obligatoria para las personas de 80 años, la que se extendió en el mes de mayo a las personas de 75 años. Esta medida prohibía la libre circulación de las personas mayores en todo el territorio nacional, por lo que el llamado era a «solo salir si es estrictamente necesario». De acuerdo con el director del Servicio Nacional del Adulto Mayor, esta medida se tomó con la finalidad de evitar el riesgo de contagios (SENAMA, 2020). Finalmente, cuatro meses más tarde, se levantaron estas restricciones, por lo que las personas mayores de 75 años quedaban sujetas a las mismas restricciones y libertades que cualquier persona, dependiendo de la fase del plan «Paso a paso» que estuviera la comuna donde residían (MINSAL, 2020c).

A pesar de las diferencias existentes entre los países del mundo, la evidencia sugiere que, hasta ahora, existen patrones similares en cuanto a contagios por COVID-19: han existido dos «olas» o peak de casos (Iftime et al., 2020). En el caso de Chile, la primera - y mayor - se desarrolló en el periodo de mayojunio en 2020, mientras que la segunda fue en enero de 2021, durante el verano. En cuanto al total de casos diagnosticados, al 23 de febrero de 2021 ascendían a 805 317. Esto significa que la transmisión actual de COVID-19 en el país es de tipo «transición comunitaria» según la OMS; es decir, que no se pueden confirmar casos a través de cadenas de transmisión. Comparado con los países vecinos, Chile presenta 4200 casos confirmados cada 100000 habitantes, cifra mayor que la del Perú y Bolivia, pero menor que la de Argentina, que presenta 4579 casos (WHO, 2021). Finalmente, los fallecidos a febrero de 2021 en Chile por COVID-19 ascienden a 20151 personas (Gobierno de Chile, 2021b).

\section{MARCO REFERENCIAL}

\subsection{Emergencias sanitarias, autoridades y medios de comunicación masivos}

En emergencias sanitarias, las autoridades y los medios de comunicación masivos juegan un rol crucial en la difusión de la información confiable y segura al público, que se denomina comunicación del riesgo. Esta incluye una gama de acciones: capacidad de comunicar para la preparación, respuesta y recuperación del evento; fomentar la toma informada de decisiones, $y$, en caso necesario, provocar cambios en el comportamiento, manteniendo la confianza en las autoridades. Sumado a esto, se encuentran grandes cambios en las sociedades modernas que influyen en la comunicación del riesgo: la pérdida de confianza en las autoridades, el hecho 
de que la mayoría del público general busca información sobre salud en fuentes online y redes sociales y el nuevo funcionamiento del «periodismo 24 horas» (Gamhewage, 2014).

De hecho, reconociendo la importancia de los medios de comunicación en la comunicación del riesgo en las emergencias sanitarias, la Organización Mundial de la Salud ha creado diversas guías dirigidas especialmente a periodistas, las cuales hacen hincapié en que la necesidad de que el público se informe desde fuentes confiables no es una función únicamente de las autoridades, sino también de los periodistas y los medios de comunicación (Dickson, 2005).

\subsection{Representaciones sociales y la esfera pública}

En un grupo de personas, es necesaria la creación de ideas colectivas para dar sentido a la realidad. Las representaciones sociales son un grupo de valores, ideas y prácticas creadas colectivamente que permiten a los miembros de una sociedad navegar a través de la vida diaria. Estas representaciones sociales poseen diversos significados, y se caracterizan por ser dinámicas, pues se construyen y reconstruyen en función de los diversos contextos históricos y socioculturales en los cuales los individuos están inmersos (Moscovici, 2008). Por lo tanto, las representaciones sociales constituyen parte del «corazón colectivo que es la opinión pública» (Villarroel, 2007, p. 443).

La opinión pública es creada dentro de la esfera pública. Habermas (1974) propone que la esfera pública es el nexo que existe entre la vida pública y la sociedad cívica, el cual emerge como un espacio social neutro, donde la opinión pública es creada. En este lugar, las autoridades juegan un rol clave, porque las percepciones que tengan las personas que ejercen cargos públicos tienen directa relación con las temáticas que eligen priorizar e implementar (Ayalon y TeschRömer, 2018). Por esta razón, es de suma importancia considerar los discursos que se generan en las diferentes instituciones públicas.

Complementando lo anterior, los medios de comunicación ejercen un rol como facilitadores de la democracia, otorgando a las personas acceso equitativo a la información, y oportunidades para participar en el debate de estas ideas. Por ende, los medios de comunicación masivos no solo cumplen el rol de informar, sino que también influyen en la formación, expresión y consumo de la opinión pública (Livingstone y Lunt, 1994).

Por lo tanto, la propiedad de los medios de comunicación es clave para entender si realmente existe libertad de expresión en estos medios. En su estudio 
sobre los medios de comunicación masivos en Chile, Ruiz-Tagle (2011) menciona que existe una concentración en cuanto a la propiedad de estos medios, donde solo algunos conglomerados concentran la propiedad de los medios de prensa escrita, televisión y radio, lo que crea que los medios de comunicación masivos sean altamente centralizados.

\subsection{Personas mayores en medios de comunicación}

La representación de las personas mayores en medios de comunicación cumple una doble función: informar, y reflejar la visión que el público en general tiene sobre ellas. A grandes rasgos, estas investigaciones han encontrado perspectivas paternalistas (Koskinene, Salminen y Leino-Kilpi, 2014), un grupo vulnerable y homogéneo, dependiente y con poca capacidad de tomar sus propias decisiones (Angulo-Giraldo et al. 2020). Estos prejuicios hacia un grupo etario es lo que Butler (1969) denominó ageism (en español se conoce como «viejismo»). Esta discriminación se manifiesta en actitudes, comportamientos, prácticas institucionales y políticas hacia las personas mayores (Butler, 1980) y puede ser encontrada en medios de comunicación, redes sociales (Makita et al., 2019), en la creación de avatares (Carrasco et al., 2017), y en la selección y ejecución de estrategias para este grupo etario (Lemaire, Brun y Régner, 2018). Por lo tanto, sus consecuencias se pueden manifestar en niveles individuales, meso y macrosociales (Ayalon y Tesch-Römer, 2018).

En cuanto a cómo se originan estas actitudes negativas, existen teorías que abordan el tema desde perspectivas micro, meso y macrosociales. En el ámbito individual, Palmore (2000) afirma que, como el envejecimiento es percibido como una pérdida de las habilidades y funcionamiento, genera una connotación negativa del proceso, a pesar de que sea inherente a los seres vivos. En el plano mesosocial, Riley y Riley (1994) sugieren que grupos, organizaciones y otras entidades sociales a través de la segregación por edad que existe en las diferentes sociedades occidentales pueden promover el viejismo, pues existe una suerte de «guion predeterminado» que establece cuáles actividades son apropiadas para cada etapa de la vida: educación, creación de una familia y trabajo, y jubilación. Finalmente, en un nivel macro, Stypińska y Nikander (2018) plantean que existen ideas culturales que discriminan a las personas mayores, porque en las sociedades modernas, a medida que las personas envejecen, pierden su poder y estatus frente a las nuevas generaciones. 
Considerando lo anterior, el principal problema del viejismo es, precisamente, reconocerlo: como hemos sido expuestos durante todas nuestras vidas a estos estereotipos negativos sobre las vejeces y el envejecer, no somos capaces de identificarlo, por lo que sus efectos suelen pasar desapercibidos (Angus y Reeve, 2006).

\section{Framing análisis}

Una de las formas de analizar los contenidos de las noticias es a través del framing analysis. Este concepto, acuñado por Erving Goffman (1974), establece que, como seres humanos, clasificamos, organizamos e interpretamos nuestras experiencias de vida para poder darles un sentido; así, creamos frames (marcos, en español) que nos ayudan a clasificar, percibir, e identificar diferentes experiencias. Complementario a esto, Gamson y Modigliani (1987), sugieren que estos marcos nos ayudan a organizar ideas y tramas. De esta forma, un análisis de noticias que utiliza este modelo se centra en estas clasificaciones que los medios crean para describir situaciones (Pan y Kosicki, 1993). Por tanto, un encuadre noticioso considera dos pasos: primero se selecciona el texto a analizar, y luego se confiere el ángulo con el que es presentado (Fernández y Antón, 2018).

Según el grupo que se esté analizando, los frames que se utilicen para su representación depende del contexto. En el caso de las personas mayores en la prensa durante la pandemia por COVID-19, estudios de diferentes países sugieren que las personas mayores han sido retratadas como un grupo homogéneo, asociado con mayores tasas de mortalidad y altamente vulnerables, que necesitan de la ayuda del resto de la sociedad (Angulo-Giraldo et al., 2020; Bravo-Segal y Villar, 2020). Por estas razones, esta investigación busca identificar los «marcos» que han sido utilizados por el Ministerio de Salud chileno para describir a las personas mayores en el contexto de pandemia.

\section{METODOLOGÍA}

Para el análisis de noticias, se utilizó la página web del MINSAL: $\underline{\text { www.minsal.cl, }}$ específicamente la sección de «destacados», que incluye noticias preparadas por un equipo periodístico destinado específicamente a esa función. Para la selección de noticias, se utilizaron tres criterios de inclusión:

- Rango de publicación: Desde el 3 de marzo de 2020, cuando se diagnosticó el primer caso de COVID-19 en Chile, hasta el 31 de diciembre de 2020. 
- Palabras clave: Persona mayor, adulto mayor o tercera edad, que podían estar en el título o en el cuerpo de la noticia. Se seleccionaron estas palabras, pues son las más comunes en Chile para referirse a las personas mayores de sesenta años (Sociedad de Geriatría y Gerontología, 2015).

- Temática: Noticias debían incluir la temática de la pandemia por COVID-19, pero también podían incorporar otras temáticas.

La búsqueda inicial originó un total de ochocientas noticias, de las cuales 105 cumplían con todos los criterios de inclusión. Luego, estas noticias fueron traspasadas al software Atlas.ti versión 7, donde se consideró cada noticia como unidad de análisis, y se codificaron manualmente. En total, se generaron cuatro familias de códigos, las cuales se detallan a continuación:

Tabla 1. Familias de códigos utilizadas

\begin{tabular}{|c|c|l|}
\hline $\begin{array}{c}\text { Familia de } \\
\text { códigos }\end{array}$ & Códigos que contiene & \multicolumn{1}{c|}{ Descripción } \\
\hline Edad & $\begin{array}{c}60 \text { a } 65 \text { años } \\
70 \text { a } 75 \text { años } \\
75 \text { a } 80 \text { años } \\
\text { Mayores de } 80 \text { años }\end{array}$ & $\begin{array}{l}\text { Edades mencionadas en las noticias. Los } \\
\text { rangos etarios se crearon en base a la infor- } \\
\text { mación presente en las noticias }\end{array}$ \\
\hline Término & $\begin{array}{c}\text { Abuelita/o } \\
\text { Abuelos } \\
\text { Adulto/s Mejor/es } \\
\text { Adulto/s mayor/es } \\
\text { Persona/s mayor/es } \\
\text { Tercera edad }\end{array}$ & $\begin{array}{l}\text { Términos con los que se referían a las } \\
\text { personas mayores de 60 años }\end{array}$ \\
\hline $\begin{array}{c}\text { Grupo de } \\
\text { riesgo }\end{array}$ & $\begin{array}{c}\text { Grupo de riesgo } \\
\text { Fragilidad } \\
\text { Grupo prioritario }\end{array}$ & $\begin{array}{l}\text { Referencia a que las personas mayores deben } \\
\text { ser consideradas un grupo prioritario por } \\
\text { sobre otros y/o que son un grupo de mayor } \\
\text { riesgo de contagio o de desarrollar la enfer- } \\
\text { medad de forma grave }\end{array}$ \\
\hline $\begin{array}{c}\text { Solida- } \\
\text { ridad } \\
\text { social }\end{array}$ & $\begin{array}{c}\text { Solidaridad social } \\
\text { Responsabilidad } \\
\text { social }\end{array}$ & $\begin{array}{l}\text { Frases que aluden al contrato social implícito } \\
\text { en las sociedades modernas sobre el cuidado } \\
\text { de las personas mayores, donde las genera- } \\
\text { ciones más jóvenes deben hacerse cargo de } \\
\text { las personas mayores, por el aporte que ellos } \\
\text { ya realizaron a la sociedad (Hashimoto, 1994) }\end{array}$ \\
\hline
\end{tabular}




\section{RESULTADOS}

En cuanto a la distribución de las noticias seleccionadas para análisis $(\mathrm{n}=108)$, la mayoría se concentra en los primeros tres meses de la pandemia en Chile: marzo, abril y mayo 2020. El gráfico 1 contiene la frecuencia de las menciones a las personas mayores de sesenta años en el periodo establecido entre el 3 de marzo de 2020 al 31 de diciembre de 2020:

\section{Gráfico 1. Frecuencia noticias sobre personas mayores}

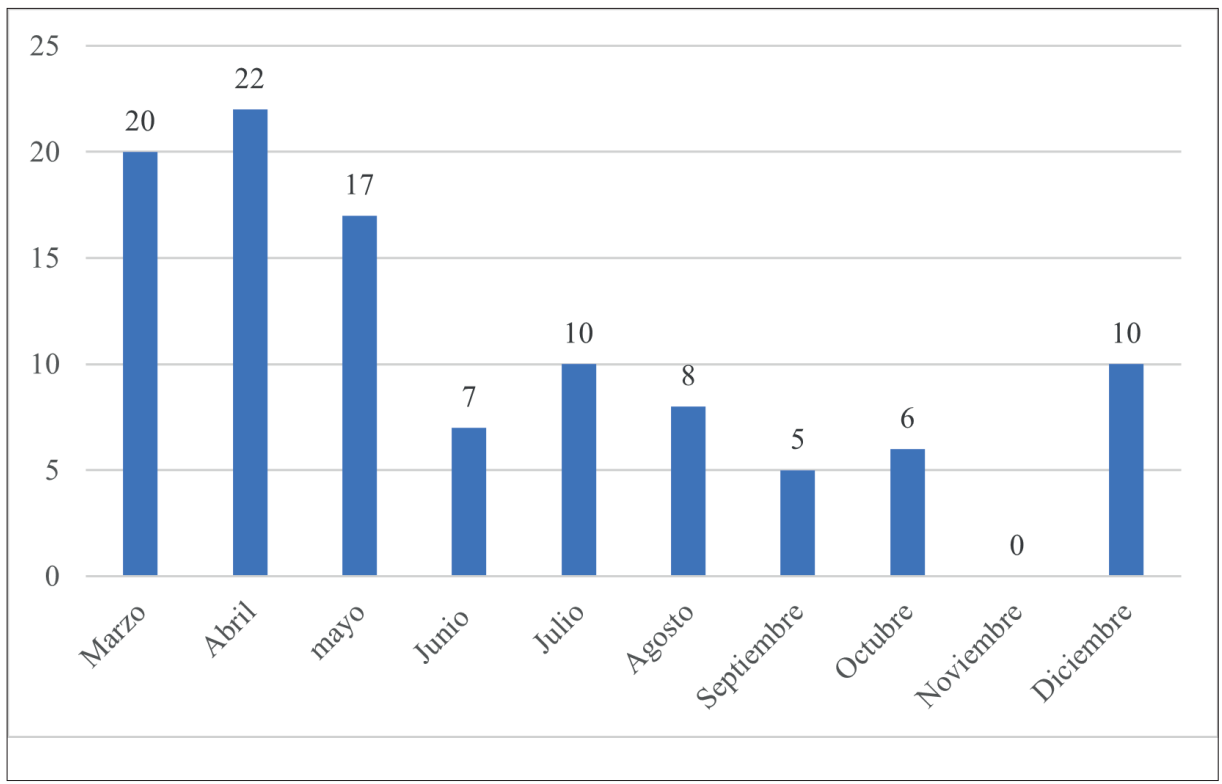

Sin embargo, luego existe un descenso, y en diciembre - mes en el que comienzan a llegar las vacunas contra el COVID-19 al país - nuevamente hay un incremento en las menciones, puesto que las personas mayores fueron consideradas como un grupo prioritario para recibir la vacuna. Sin embargo, llama la atención que, durante el mes de noviembre, no se encontraron noticias que incluyeran a la población adulta mayor.

\subsection{Subgrupos etarios}

Como se mencionó anteriormente, en Chile, desde el punto de vista legal, son consideradas personas mayores las personas desde los sesenta años; sin embargo, 
este grupo etario a su vez contiene varios subgrupos. El gráfico 2 contiene información sobre los subgrupos etarios mencionados en las noticias:

\section{Gráfico 2. Subgrupos etarios}

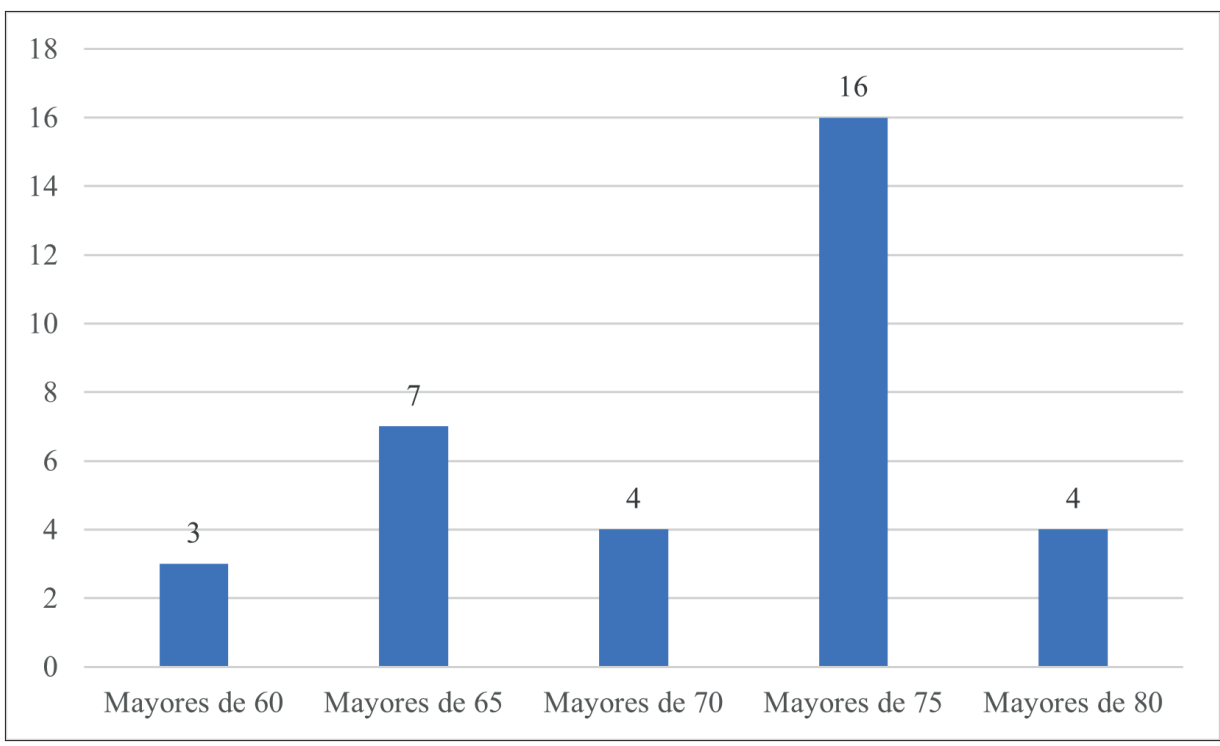

Del total de las 34 menciones que se realizaron sobre la edad de las personas mayores, la mayor frecuencia $(n=16)$ se encuentra en el grupo de mayores de 75 , esto es porque gran parte de las medidas que tomó el gobierno durante la pandemia por COVID-19, se centraron en este grupo en particular (MINSAL, 2020b).

\subsection{Términos para referirse a las personas mayores}

En cuanto a los términos utilizados para referirse a las personas mayores de sesenta años, se encontraron 204 menciones, entre las que se identificaron seis términos, cuya frecuencia por orden alfabético se encuentra en el gráfico 3:

El término más frecuente utilizado en las noticias fue adultos mayores $(\mathrm{n}=143)$, que corresponde a la forma en que este grupo etario es representado en la Ley N 19.828 de 2002. Sin embargo, en el año 2018, el MINSAL publicó una guía donde establece que el término «persona/s mayor/es» es el que se debe utilizar. Por esta razón, el segundo término con mayor frecuencia $(n=47)$ es personas mayores. 


\section{Gráfico 3. Frecuencia términos personas mayores}

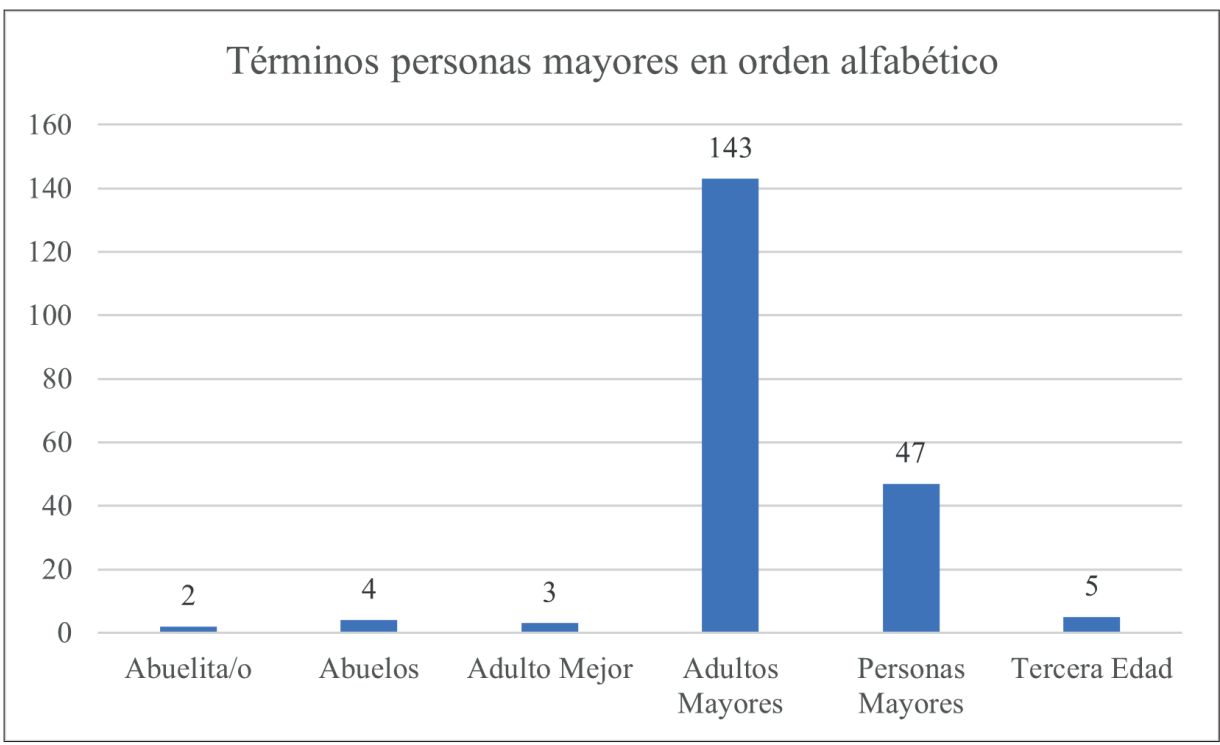

\subsection{Grupo de riesgo}

En las noticias, las personas mayores también fueron referidas como un grupo que tenía mayor riesgo de contraer la enfermedad o de desarrollar consecuencias graves que las llevaran a necesitar ventilación mecánica (MINSAL, 15 de marzo). Esta familia de códigos está conformada por tres subcódigos: grupo de riesgo $(\mathrm{n}=92)$, grupo prioritario $(\mathrm{n}=6)$ y fragilidad $(\mathrm{n}=5)$.

Las menciones que presentan a las personas mayores como un grupo de riesgo o frágil incluían cuñas directas de las autoridades, como la que dijo el presidente Sebastián Piñera el 22 de marzo de 2020 durante un discurso: «proteger la salud y la vida de todos, y muy especialmente la de nuestros adultos mayores, que son los que experimentan los mayores riesgos» (MINSAL, 2020d), así como también en la redacción de la noticia, por el equipo periodístico el 23 de marzo: «Con respecto a las personas de la tercera edad, quienes son el principal grupo de riesgo de desarrollar la enfermedad COVID-19 de forma severa» (MINSAL, 2020e). Paralelamente, las autoridades hacían alusión a la responsabilidad de los grupos más jóvenes en el contagio de la enfermedad, como dijo el ministro de Salud, Enrique Paris, el 7 de diciembre de 2020: «Y esto quiero que lo escuchen muy bien las personas jóvenes. Es evidente que el mayor motor de contagio está 
entre los 20 y 40 años edad y vemos que es la población que menos se cuida y puede llevar el virus a los adultos mayores» (MINSAL, 2020f).

A esto se suman las referencias a las personas mayores de 65 años como un grupo prioritario, sobre todo en cuanto a la aplicación de vacunas. En el periodo analizado, las noticias del MINSAL también se referían a la vacunación contra la influenza estacional. Por lo tanto, aparecían menciones a este grupo etario como preferente para recibir estas vacunas. Más tarde, desde el 22 de septiembre de 2020, inician las alusiones a la adquisición y posterior aplicación de la vacuna contra el COVID-19: «El Presidente detalló que, una vez que haya una vacuna segura y eficaz, será necesario en primer lugar vacunar a la población que es más vulnerable al COVID-19. [...] Esta prioridad incluye a los trabajadores de la salud, los mayores de 65 años, los enfermos crónicos y las personas en centros cerrados, para luego seguir por rango etario hasta llegar a los más jóvenes (MINSAL, 2020g).

\subsection{Solidaridad social}

Desde la categoría anterior, se desprende la solidaridad o responsabilidad social. Partiendo desde la premisa de que la mayoría de las personas mayores, a pesar de ser autónomas, necesitarán algún tipo de asistencia durante sus vidas (Sulbrandt, Pino y Oyarzún, 2012), Bengtson y Achembaum (1993) sugieren que los recursos disponibles para asistir o cuidar a las personas mayores son limitados, por lo que existe una suerte de organización estructural que requiere un contrato implícito entre las generaciones que establece quiénes necesitan el cuidado (personas mayores) y quiénes deben ser los cuidadores (generaciones jóvenes). Este contrato simbólico entre generaciones se basa en la solidaridad intergeneracional. Entonces, tomando como base la categoría anterior — donde se evidencia que para el ministerio las personas mayores durante la pandemia han sido consideradas como un grupo de riesgo-, en reiteradas ocasiones, autoridades de diversas instituciones hacían el llamado a «cuidar» a las personas mayores: hay un total de 42 referencias a este tema.

Por ejemplo, el 18 de marzo de 2020 el presidente de la república mencionó: «Generosidad y no egoísmos. Tranquilidad y disciplina para enfrentar esta pandemia. Cuidemos nuestra salud. Y también cuidemos la salud de los demás, y muy especialmente, la de nuestros adultos mayores» (MINSAL, 2020h). Ese mismo mes, el entonces ministro de Salud, Jaime Mañalich, hacía un llamado a que «las personas deben tomar distancia para así cuidar a nuestros adultos mayores 
que representan el segmento de población más frágil para esta enfermedad». (MINSAL, 2020i). Más tarde, el 16 de abril, el subsecretario de Redes Asistenciales, Arturo Zúñiga, reiteraba el llamado a «la ciudadanía a cuidar de nuestros padres y de nuestros abuelos. Son las personas mayores las que están en mayor riesgo ante esta pandemia, por lo que su cuidado debe ser fundamental». Paralelamente, se encuentra en algunas de estas frases el uso de adjetivos posesivos para referirse a este grupo etario, como «nuestros» adultos mayores y «nuestros» abuelos (MINSAL, 2020j).

Asimismo, existen referencias a que las mujeres son las que mayoritariamente se hacen cargo del cuidado de los otros en Chile, especialmente de las personas mayores. En en octubre de 2020, la subsecretaria de Salud Pública declaró que «las mujeres se han dedicado principalmente en este período a tareas domésticas, cuidado de los hijos, también a cuidar a los adultos mayores. Por eso, las invitamos a hacer un párele. Paren y preocúpense de ustedes» (MINSAL, 2020k).

\section{DISCUSIÓN}

Del universo de 108 noticias analizadas desde el sitio web del Ministerio de Salud de Chile (MINSAL), fue posible encontrar cuatro «marcos» que fueron utilizados para referirse a las personas mayores en el contexto de pandemia durante el año 2020: subgrupos etarios, términos, grupos de riesgo y solidaridad social. Como ya se mencionó, estas representaciones sociales son claves, pues son aspectos compartidos socialmente e influyen en la percepción que tanto las autoridades como el público general se forma sobre las personas mayores de sesenta años.

Con respecto al primer marco, sobre el rango etario con el que se referían las noticias a las personas mayores, el que concentra la mayor frecuencia es el de 75 años y más, porque en particular, existieron diversas medidas que estaban específicamente dirigidas a ese grupo, como la opción de teletrabajo para los funcionarios públicos que inició en marzo, o la cuarentena obligatoria en el país.

La medida de establecer cuarentenas especialmente dirigidas a las personas mayores ha sido cuestionada especialmente por los efectos que los confinamientos obligatorios/voluntarios han provocado en la población; se considera que «han propiciado un deterioro gradual de la salud física y mental de las personas mayores» (Cepal, 2020). Muchas personas no pudieron ver a sus familias y amigos durante este tiempo, debilitando sus redes de apoyo. Esto afectó especialmente a las personas mayores residentes de los ELEAM, porque durante ese tiempo se prohibieron las visitas. Además, los niveles de sedentarismo aumentaron, porque 
las posibilidades de realizar actividades físicas par este grupo disminuyeron debido a las restricciones. El sedentarismo, sumado la exclusión social, se ha documentado que constituyen un mayor riesgo de enfermedades no transmisibles, especialmente durante la pandemia por COVID-19 (Bouillon-Miniois, Lahaye y Dutheil, 2020; Plagg et al., 2020). De hecho, un estudio realizado en Santiago de Chile (Observatorio del Envejecimiento, 2020) evidenció que un 45\% de las personas mayores consideraba que su salud mental había empeorado, y que un $24 \%$ decía estar desanimado o muy desanimado.

Por otro lado, entre los términos utilizados por el MINSAL para referirse a las personas mayores, «adulto mayor» surge como el que concentra la mayor frecuencia, tanto en el cuerpo de las noticias, como en las citas de autoridades, a pesar de que el ministerio en el año 2018 publicó la guía «Nuevas expresiones para referirse a las personas mayores», para disminuir el estigma asociado con la vejez y el envejecimiento. Esta establece que se debe priorizar el concepto «persona mayor» para referirse a las personas sobre sesenta años, porque el concepto «adulto mayor» fue cambiado al adoptar el enfoque de género (MINSAL, 2018). De hecho, las únicas autoridades que utilizaron el concepto «persona mayor» fueron la subsecretaria de Salud Pública, el director del Servicio Nacional del Adulto Mayor (SENAMA) y especialistas de la Sociedad de Geriatría y Gerontología de Chile.

Sin embargo, esta forma de referirse a este grupo etario evidencia una falta de enfoque de género en la forma en que se describe a las personas mayores, ignorando un importante aspecto de la vejez, que es la variedad de experiencias en esta etapa de la vida. De hecho, la evidencia sugiere que, dentro del grupo de personas mayores, las mujeres son las que presentan mayores dificultades en diferentes aspectos de sus vidas, debido a la acumulación de desigualdades de las que han sido víctimas durante toda su vida (Dannefer, 2003; Ferraro y Shippee, 2009). En el caso de Chile, las mujeres que actualmente son adultas mayores fueron criadas en un contexto sociocultural muy diferente, donde las posibilidades de acceso a la educación formal eran reducidas por el solo hecho de ser mujeres.

Otra forma de representar a las personas mayores en las noticias en el sitio web era la de un grupo de riesgo; es decir, que era un grupo que debía ser cuidado por el resto, lo que además se relaciona con el último marco identificado: solidaridad/responsabilidad social. Ambas formas de representar a las personas mayores implican que son percibidas en la esfera pública como un grupo que no tiene autonomía, y que para conllevar la pandemia necesita del resto. Esto coincide con hallazgos de previas investigaciones realizadas en Chile, donde el cuidado 
de las personas mayores dependientes está ligado especialmente a las mujeres, por asociar roles tradicionales de género, donde se asocia a las mujeres como cuidadoras naturales, que poseen características que las calificarían mejor para cumplir esta función (Salazar, 2017; Osorio-Parraguez, Navarrete y Briones, 2019).

De hecho, se puede observar que gran parte de las medidas promovidas por las autoridades chilenas en el contexto de pandemia de COVID-19 está focalizada hacia el cuidado colectivo en lugar de hacer un llamado al autocuidado. Por esta razón, las autoridades hicieron constantes referencias a los otros grupos etarios de la sociedad, que podían ayudar o perjudicar a las personas mayores. En este caso, se encontró que la mayoría de las menciones responsabilizaban especialmente a los jóvenes como el grupo que podía contagiar a las personas mayores (MINSAL, 2020f), lo que coincide con los hallazgos de un estudio de la prensa chilena, en el contexto de pandemia, donde se retrataba a los jóvenes como un grupo social «peligroso para los demás, sobre todo para las y los adultos mayores» (Araya, Carrasco y Olivares, 2020, p. 24).

Por último, otro aspecto importante a considerar de las noticias analizadas es que la mayoría se basa en hechos ocurridos en la Región Metropolitana, donde se encuentra Santiago, la capital del país. Efectivamente, los medios de comunicación chilenos han sido tildados de "centralistas», puesto que la capital concentra la atención mediática (Hofflinger, 2020) y las experiencias de personas de otras zonas son invisibilizadas.

\section{CONCLUSIONES}

Este artículo analizó cómo el Ministerio de Salud de Chile retrató en sus noticias a las personas mayores de sesenta años durante el año 2020 en el contexto de pandemia por COVID-19: se identificaron cuatro marcos que evidenciaban discriminación por edad: se representa a las personas mayores como un grupo homogéneo, que depende del resto de la sociedad para poder conllevar la pandemia, a pesar de que el sitio web analizado pertenece al MINSAL, cuya principal función es informativa. Es llamativo el hecho de que, del universo de 108 notas de prensa analizadas, solo dos (publicadas el 18 de abril y 15 de julio de 2020, respectivamente) contenían cuñas directas desde las personas mayores, mientras que el resto son otras personas hablando sobre este grupo etario. Sin embargo, es importante notar que esta ausencia de cuñas directas puede ser resultado de que, al tiempo de realizarse estas notas, había muchas restricciones 
sanitarias vigentes que limitaban el contacto con las personas mayores, especialmente las mayores de setenta y cinco años.

De todas formas, contar con la experiencia directa desde los actores - en este caso, las personas mayores - ayudaría a visibilizar los reales efectos que ha provocado la pandemia en este grupo que, según Wasiek (2021), ha sido invisibilizado, porque gran parte de la cobertura que se ha realizado ha estado enfocada a presentar las estadísticas, más que la percepción cualitativa de las personas.

Más que nunca, en el contexto de pandemia, se vuelve muy importante garantizar los derechos de las personas mayores, pero esto no implica necesariamente adoptar un enfoque paternalista e infantilizador que presente a las personas mayores como un grupo sin agencia. De hecho, en el último tiempo, los modelos sobre un buen envejecer, se han movido desde una extrema independencia —como el «envejecimiento exitoso» que Rowe y Khan (1987) planteaban - a un modelo de envejecimiento armonioso (Liang y Luo, 2012), donde exista un balance entre las pérdidas y ganancias propias de esta etapa de vida. Por otro lado, Lamb (2014) propone que un buen envejecer debe basarse en parte, en asumir que interdependencia es inherente a los seres humanos, pero que simultáneamente se debe respetar la autonomía que tienen las personas mayores.

Por lo mismo, la representación que realizan las autoridades y los medios de comunicación de las personas mayores es de suma importancia, pues si estas son negativas, contribuye a las perspectivas viejistas en la esfera pública que han sido documentadas previamente, como que la vejez es un problema que debe ser solucionado. Por esta razón, es de vital importancia el lenguaje que se utiliza al referirse a las vejeces y el envejecimiento (Lundebjerg, Trucil, Hamond, y Applegate, 2017).

Si bien existen estudios que han analizado la representación de las personas mayores en la prensa, es necesario expandir esta área hacia otros actores claves, como las autoridades. La visión que tienen las autoridades sobre determinados grupos interferirá directamente en la planificación e implementación de políticas sociales dirigidas al grupo determinado. 


\section{REFERENCIAS}

Angulo-Giraldo, M.A., Ramos G., Chamorro, H., Colonia, L. y Fernández-Alvarado, S. (2020). Personas mayores en los medios digitales peruanos durante la pandemia por COVID-19. Kairos Gerontologia, 23, 391-416. Recuperado de https://revistas.pucsp.br/index.php/kairos/article/view/51532

Angus, J. y Reeve, P. (2006). Ageism: A threat to «aging well» in the 21st century. Journal of Applied Gerontology, 25(2), 137-152. https://doi. org/10.1177\%2F0733464805285745

Araya, C., Carrasco, P. y Olivares, J. (2020). Reflexiones sobre la visibilización de lo juvenil por la prensa escrita chilena, en contexto de pandemia. Última Década, 53, 5-39. https://doi.org/10.4067/S0718-22362020000100005

Ayalon, L., Chasteen, A., Diehl, M., Levy, B., Neupert, S., Rothermund, K., . . . Wahl, H. (2020). Aging in times of the COVID-19 pandemic: Avoiding ageism and fostering intergenerational solidarity. Journals of Gerontology: Psychological Sciences, $X X(\mathrm{XX}), 1-4$. Recuperado de https:/academic.oup.com/ psychsocgerontology/article/76/2/e49/5820621

Ayalon, L. y Tesch-Römer, C. (2018). On the manifestations and consequences of ageism. En L. Ayalon y C. Tesch-Römer (eds.), Contemporary perspectives on ageism (pp. 109-113). Nueva York: Springer. https://doi.org/10.1007/978-3319-73820-8_7

Bauer, M. W. y Gaskell, G. (2008). Social representations theory: A progressive research programme for social psychology. Journal of the Theory of Social Behaviour, 38(4), 335-508. https://onlinelibrary.wiley.com/doi/10.1111/j.14685914.2008.00374.x

Bengtson, V. L. y Achenbaum, W. A. (1993). The changing contract across generations. Nueva York: A. de Gruyter.

Bravo-Segal y Villar, F. (2020). La representación de los mayores en los medios durante la pandemia COVID-19: ¿hacia un refuerzo del edadismo? Revista Española de Geriatría y Gerontología, 55(5), 266-271. https://doi.org/10.1016/j. regg.2020.06.002

Bouillon-Minois J., Lahaye C., Dutheil F. (2020). Coronavirus and quarantine: Will we sacrifice our elderly to protect them? Archives of Gerontology and Geriatrics, 90, 1-2. https://doi.org/10.1016/j.archger.2020.104118

Butler, R. N. (1969). Age-ism: Another form of bigotry. The Gerontologist, 9(4), 243-246. https://doi.org/10.1093/geront/9.4_Part_1.243

Butler, R. N. (1980). Ageism: A foreword. Journal of Social Issues, 36(2), 8-11. https:// doi.org/10.1111/j.1540-4560.1980.tb02018.x 
Carrasco, R., Baker, S., Waycott, J. y Vetere, F. (2017). Negotiating stereotypes of older adults through avatars. Paper presented at the 29th Australian Conference on Computer-Human Interaction, 218-227. https://dl.acm.org/doi/ pdf/10.1145/3152771.3152795

Cepal (2020). Desafíos en la protección de las personas mayores y sus derechos frente a la pandemia del COVID-19. Recuperado de https://www.cepal.org/es/enfoques/ desafios-la-proteccion-personas-mayores-sus-derechos-frente-la-pandemiacovid-19

Dannefer, D. (2003). Cumulative advantage/disadvantage and the life course: Crossfertilizing age and social science theory. The Journals of Gerontology: Series B, 58(6), S327-S337. https://doi.org/10.1093/geronb/58.6.S327

Dickson, D. (2005). Bird flu: The role of science journalists. WHO. Recuperado de https://apps.who.int/iris/bitstream/handle/10665/69203/WHO_CDS_2005.37_ eng.pdf? sequence $=1 \&$ isAllowed $=\mathrm{y}$

Fernandez, M. y Anton, M. (2018). Estereotipos de las personas mayores y de género en la prensa digital: Estudio empírico desde la teoría del framing. Revista Prisma Social, 316-337. Recuperado de https://revistaprismasocial.es/article/view/2443

Ferraro, K. F. y Shippee, T. P. (2009). Aging and cumulative inequality: How does inequality get under the skin? The Gerontologist, 49(3), 333-343. https://doi. org/10.1093/geront/gnp034

Gamson, W.y Modigliani,A.(1989). Media discourse and public opinion:Aconstructionist approach. American Journal of Sociology, 97, 1-37. https://doi. org/10.1086/229213

Gamhewage, G. (2014). An introduction to risk communication. WHO. Recuperado de https://www.who.int/risk-communication/introduction-to-risk-communication. pdf?ua $=1$

Gobierno de Chile (2021a). Paso a paso. Recuperado de https://www.gob.cl/coronavirus/pasoapaso/

Gobierno de Chile (2021b). Cifras oficiales COVID-19. Recuperado de https://www. gob.cl/coronavirus/cifrasoficiales/

Goffman, E. (1974). Frame analysis: An essay on the organization of experience. Nueva York: Harper / Row.

Habermas, J. (1974). The public sphere: An encyclopedia article. New German Critique, 3, 49-55. https://doi.org/10.2307/487737

Hashimoto, A. (1996). The gift of generations: Japanese and american perspectives on aging and the social contract. Cambridge; Nueva York: Cambridge University Press. https://doi.org/10.1017/CBO9780511571077 
Hofflinger, A. (2020). COVID-19 y el centralismo de los medios de comunicación. Recuperado de https://www.ciperchile.cl/2020/05/04/covid-19-y-el-centralismode-los-medios-de-comunicacion/

Iftime, A., López-Azcona, I., Hernandez-Flix, S., de Febrer, G., Parra, S., HernándezAguilera, A., Riu, F., .. . Castro, A. (2020). First and second waves of coronavirus disease-19: A comparative study in hospitalized patients in Reus, Spain. MedRxiv, 1-21. https://doi.org/10.1101/2020.12.10.20246959

Kang, S. y Jung, S. I. (2020). Age-related morbidity and mortality among patients with COVID-19. Infection \& Chemotherapy, 52(2), 154-164. https://doi.org/10.3947/ ic.2020.52.2.154

Koskinene, S., Salminen, L. y Leino-Kilpi, H. (2014). Media portrayal of older people as illustrated in finnish newspapers. International Journal of Qualitative Journal of Qualitative Studies on Health and Well-Being, 9(1), 1-11. https://doi. org/10.3402/qhw.v9.25304

Lamb, S. (2014). Permanent personhood or meaningful decline? Toward a critical anthropology of successful aging. Journal of Aging Studies, 29(0), 41-52. https:// doi.org/10.1016/j.jaging.2013.12.006

Lemaire, P., Brun, F. y Regner, I. (2018). Negative aging stereotypes disrupt both the selection and execution of strategies in older adults. Gerontology, 64, 373-381. https://doi.org/10.1159/000486756

Liang, J. y Luo, B. (2012). Toward a discourse shift in social gerontology: From successful aging to harmonious aging. Journal of Aging Studies, 26(3), 327-334. https://doi.org/10.1016/j.jaging.2012.03.001

Livingstone, S. y Lunt, P. (1994). The mass media, democracy and the public sphere. En S. Livingstone y P. Lunt (eds.), Talk on television audience participation and public debate (pp. 9-35). Londres: Routledge.

Lundebjerg, N., Trucil, D., Hamond, E. y Applegate, W. (2017). When it comes to older adults, language matters: Journal of the American Geriatrics Society adopts modified american medical association style. Journal of the American Geriatrics Society, 65(7), 1386-1388. https://doi.org/10.1111/jgs.14941

Makita, M., Mas-Bleda, A., Stuart, E. y Thelwall, M. (2019). Ageing, old age and older adults: A social media analysis of dominant topics and discourses. Ageing \& Society. https://doi.org/10.1017/S0144686X19001016

Mejía, C., Rodríguez-Alarcón, F., Garay-Ríos, L., Enríquez-Anco, M. d. G., Moreno, A., Huaytan-Rojas, K., . . Curioso, W. (2020). Percepción de miedo o exageración que transmiten los medios de comunicación en la población peruana durante la pandemia de la COVID-19. Revista Cubana de Investigaciones Biomédicas, 
39(2), 1-20. Recuperado de http://scielo.sld.cu/scielo.php?script=sci_arttext\&pi $\mathrm{d}=\mathrm{S} 0864-03002020000200001$

Menéndez, E. (1994). La enfermedad y la curación. ¿Qué es medicina tradicional? Alteridades, 4(7), 71-83. Recuperado de https://www.redalyc.org/ pdf/747/74711357008.pdf

Menéndez, E. (2009). De sujetos, saberes y estructuras: Introducción al enfoque relacional en el estudio de la salud colectiva. Buenos Aires: Lugar Editorial.

MINSAL (2018). Nuevas expresiones para referirse a las personas mayores. Recuperado de https://www.minsal.cl/wp-content/uploads/2018/09/NUEVAS-EXPRESIONES-PARA-REFERIRSE-A-LAS-PERSONAS-MAYORES-PDF.pdf

MINSAL (2020a). Ministerio de Salud confirma primer caso de coronavirus en Chile. Recuperado de https://www.minsal.cl/ministerio-de-salud-confirmaprimer-caso-de-coronavirus-en-chile/

MINSAL (2020b). Presidente Sebastián Piñera anunció medidas para enfrentar el covid-19. Recuperado de https://www.minsal.cl/presidente-sebastian-pineraanuncio-medidas-para-enfrentar-el-covid-19/

MINSAL (2020c). Gobierno levanta restricciones para desplazamiento de adultos mayores de 75 años. Recuperado de https://www.minsal.cl/gobierno-levantarestricciones-para-desplazamiento-de-adultos-mayores-de-75-anos/

MINSAL (2020d). Presidente Piñera anuncia medida de protección para adultos mayores. Recuperado de https://www.minsal.cl/presidente-pinera-anuncia-medida-de-proteccion-para-adultos-mayores/

MINSAL (2020e). Ministro de Salud anunció más de mil habitaciones de aislamiento en el país. Recuperado de https://www.minsal.cl/ministro-de-salud-anunciomas-de-mil-habitaciones-de-aislamiento-en-el-pais/

MINSAL (2020f). COVID-19: Todas las comunas de la Región Metropolitana retroceden a Transición a partir de este jueves 10 de diciembre. Recuperado de https:// www.minsal.cl/covid-19-todas-las-comunas-de-la-region-metropolitana-retroceden-a-transicion-a-partir-de-este-jueves-10-de-diciembre/

MINSAL (2020g). Presidente Piñera anuncia avances para la obtención de potenciales vacunas contra el COVID-19. Recuperado de https://www.minsal.cl/ presidente-pinera-anuncia-avances-para-la-obtencion-de-potenciales-vacunascontra-el-covid-19/

MINSAL (2020h). Presidente declara Estado de Excepción Constitucional de Catástrofe en todo el territorio nacional. Recuperado de https://www.minsal.cl/ presidente-declara-estado-de-excepcion-constitucional-de-catastrofe-en-todoel-territorio-nacional/ 
MINSAL (2020i). Ministerio de Salud reporta 2449 casos de COVID-19 en el país. Recuperado de https://www.minsal.cl/ministerio-de-salud-reporta-2-449-casosde-covid-19-en-el-pais/

MINSAL (2020j). Minsal anuncia que uso de mascarilla en lugares públicos cerrados será obligatorio. Recuperado de https://www.minsal.cl/minsal-anuncia-que-usode-mascarilla-en-lugares-publicos-cerrados-sera-obligatorio/

MINSAL (2020k). COVID-19: Positividad de exámenes PCR disminuye por primera vez a 3\% en RM. Recuperado de https://www.minsal.cl/covid-19-positividad-deexamenes-pcr-disminuye-por-primera-vez-a-3-en-rm/

Moscovici, S (2008). Psychoanalysis: Its Image and its Public. Cambridge: Polity Press.

Nespereira, J. (2014). Los discursos de la pandemia. nuevas estrategias de comunicación del riesgo en un nuevo contexto sociocultural. Cultura, Lenguaje y Representación, 13, 185-199. Recuperado de https:/www.e-revistes.uji.es/index.php/clr/ article/view/1553. https://doi.org/10.6035/CLR.2014.13.10

Observatorio del envejecimiento (2020). Saludmentaly principales preocupaciones de las personas mayores en contexto de pandemia. Santiago. Recuperado de https:// observatorioenvejecimiento.uc.cl/wp-content/uploads/2020/12/Salud-Mentalen-Pndemia.pdf

Osorio-Parraguez, P., Navarrete, I. y Briones, S. (2019). Aproximación etnográfica a las manifestaciones de agencia en personas nonagenarias y centenarias en chile. Etnográfica, 23(3). https://doi.org/10.4000/etnografica.7400

Palmore, E. (2000). Guest editorial: Ageism in gerontological language. The Gerontologist, 40(6), 645-645. https://doi.org/10.1093/geront/40.6.645

Pan, Z. y Kosicki, G. (1993). Framing analysis: An approach to news discourse, 10, 55-75. https://doi.org/10.1093/geront/40.6.645

Plagg B., Engl, A., Piccoliori, G., Eisendle, K. (2020). Prolonged social isolation of the elderly during COVID-19: Between benefit and damage. Archives of Gerontology and Geriatrics. 89: 1-2. https://doi.org/10.1016/j.archger.2020.104086

Rahim, A., Ibrahim, M., Salim, F. y Ariffin, M. (2019). Health information engagement factors in malaysia: A content analysis of facebook use by the ministry of health in 2016 and 2017. International Journal of Environmental Research and Public Health, 16(4), 1-11. https://doi.org/10.3390/ijerph16040591

Riley, M. W. y Riley, J. W., Jr. (1994). Structural lag: Past and future. En M. W. Riley, R. L. Kahn y A. Foner (eds.), Age and structural lag: Society's failure to provide a meaningful opportunity in work, family, and leisure (pp. 15-36). Nueva York: Wiley.

Rowe, J. W. y Kahn, R., L. (1987). Human aging: Usual and successful. Science, 237(4811), 143-149. https://doi.org/10.1126/science.3299702 
Ruiz-Tagle P. (2011). Propiedad de los medios y principios de intervención del estado para garantizar la libertad de expresión en Chile. Revista de Derecho (Coquimbo), 18(2), 347-359. https://doi.org/10.4067/S0718-97532011000200012

Salazar, C. (2017). Social contract on elderly caregiving in contemporary Chile. Perspectives of a group of social scientists. Anthropology \& Aging, 38(1), 60-79. https://doi.org/10.5195/aa.2017.134

SENAMA (2020). Más de un millón de personas de 75 años y más inician cuarentena preventiva obligatoria a contar de este viernes. Recuperado de http://www. senama.gob.cl/noticias/mas-de-un-millon-de-personas-de-75-anos-y-masinician-cuarentena-preventiva-obligatoria-a-contar-de-

Shahid, Z., Kalayanamitra, R., McClafferty, B., Kepko, D., Ramgobin, D., Patel, R., . . . Jain, R. (2020). COVID-19 and older adults: What we know. Journal of the American Geriatrics Society, 65(5), 926-929. https://doi.org/10.1111/jgs.16472

Smith, J. L. y Bryant, F. B. (2019). Enhancing positive perceptions of aging by savoring life lessons. Aging \& Mental Health, 23(6), 762-770. https://doi.org/10.1080/13 607863.2018.1450840

Stypińska, J. y Nikander, P. (2018). Ageism and age discrimination in the labour market: A macrostructural perspective. En L. Ayalon y C. Tesch-Römer(eds.), Contemporary perspectives on ageism: Vol. 19. International Perspectives on Aging (pp. 73-90). Berlín: Springer. https://doi.org/10.1007/978-3-319-73820-8_6

Sulbrandt, J., Pino, P. y Oyarzún, M. (2012). Envejecimiento activo y saludable: Investigación y políticas para el envejecimiento poblacional. Revista Chilena de Enfermedades Respiratorias, 28(4), 269-271. https://doi.org/10.4067/S071773482012000400001

Villarroel, G. (2007). Las representaciones sociales: una nueva relación entre el individuo y la sociedad. Fermentum, 17(49), 434-454. Recuperado de https://www. redalyc.org/pdf/705/70504911.pdf

Wasiek, C. (2021). Personas mayores en tiempos de la pandemia del covid-19. Recuperado de https:/www.gerontologia.org/portal/noticia.php?id=4695

WHO (2021). WHO coronavirus disease (COVID-19) dashboard. Recuperado de https:// covid19.who.int/

Yañez, D., Weiss, N., Romand, J. y Treggiari, M. (2020). COVID-19 mortality risk for older men and women. BMC Public Health, 20(1742), 1-7. https://doi. org/10.1186/s12889-020-09826-8

Zulman, D., Sussman, J., Chen, X., Cigolle, C., Blaum, C. y Hayward, R. (2011). Examining the evidence: A systematic review of the inclusion and analysis of older adults in randomized controlled trials. Journal of General Internal Medicine, 26, 783-790. https://doi.org/10.1007/s11606-010-1629-x 